\title{
Surprising attraction of non-magnetic materials to magnets
}

\author{
Tin Vo and Gerald H. Pollack \\ Department of Bioengineering
}

Box 355061, University of Washington, Seattle WA, 98195

\section{ABSTRACT}

We explored the effects of static magnetic fields on materials commonly classified as non-ferromagnetic and non-paramagnetic. By floating pieces of paper, wood, Teflon, and Nafion on different liquids and placing those objects near a neodymium magnet, we found that the objects moved consistently towards the magnet. As the distance between object and magnet diminished, the attraction strengthened. The results imply that objects currently classified as non-magnetic may in fact be at least weakly magnetic.

Language: English

Date of Publication: 30-06-2018

DOI: 10.24297/jap.v14i2.7368

ISSN: 2347-3487

Volume: 14 Issue: 2

Journal: Journal of Advances in Physics

Website: https://cirworld.com

This work is licensed under a Creative Commons Attribution 4.0 International License. 


\section{INTRODUCTION}

Discovered well before electrical forces, magnetism is a force known to the ancients [1]. Earlier civilizations relied on this force to create devices such as compasses [2]. Magnetism is now broadly studied in many realms and with many materials, particularly with regard to practical applications [3-7].

Materials attracted to magnets are commonly classified as ferromagnetic or paramagnetic. Ferromagnetic materials, such as iron and nickel, display a strong attraction to magnets. Paramagnetic substances, like magnesium and lithium, also exhibit attraction, but weaker than that experienced by ferromagnetic objects [8]. Ferromagnetic materials retain their magnetic properties after being removed from magnetic fields, while paramagnetic substances do not.

In the current experiments, we used a sensitive system to explore various substances currently classified as neither ferromagnetic nor paramagnetic. We found that when placed in strong external magnetic fields, wood, paper, Teflon, and Nafion exhibit a positive susceptibility.

\section{METHODS}

To begin the standard experiment, $35.5 \mathrm{~mL}$ of deionized (DI) water was obtained from a Barnstead D3750 Nanopure Diamond purification system (type 1 HPLC grade $18.2 \mathrm{M} \Omega$ ) and poured into a 9.4-cm diameter glass petri dish.

For experiments involving paper, a $1 \mathrm{~mm}$ by $5 \mathrm{~mm}$ strip was cut out from standard cardstock paper, $1 \mathrm{~mm}$ thick, used for commercial packaging. The strip was placed so that it would float on the water in the petri dish. Strips were positioned away from the edges of the dish to prevent the paper from clinging. To prevent room-air currents from affecting the paper's position, a glass lid was placed over the petri dish. The setup was then left undisturbed for 2 minutes to allow any water ripples created by placing the paper on the water surface to recede.

To create the magnetic field, a $45 \times 25 \times 25 \mathrm{~mm}$ neodymium block magnet was placed on top of the glass lid near one edge of the petri dish. The south pole of the magnet faced into the dish. To record the movement of the paper, a video camera (Edmund Optics (3112C $1 / 2$-inch CMOS Color USB) was mounted above the setup. The camera was immediately turned on and continued recording at 11 frames per second until the paper had moved enough to be hidden from the camera's view.

For analysis, the video was run through video-processing software (ImageJ). This permitted analysis of the time course of paper position relative to the magnet. For calibration, the measurement in pixels was compared to the known dimension of the paper, allowing conversion of pixels into distance. The paper's distance from the magnet vs. time was recorded and plotted.

To investigate whether the effect was limited to paper alone, the strip of paper was replaced with a piece of Teflon, Nafion, or wood. In the case of Nafion and Teflon, tubes were used in lieu of flat rectangular sheets, while the wood sample was a small end segment of an ordinary toothpick.

To determine whether the observed effect might arise from some feature of the suspending medium, the water was replaced with mineral oil (Sigma-Aldrich) or ethanol (Decon Laboratories Inc.) In the case of oil, because of the reduced speed arising from its high viscosity, the magnet was placed relatively closer (13 mm vs. $30 \mathrm{~mm}$ ) to the nearest edge of the paper. This same starting separation of $13 \mathrm{~mm}$ was maintained also for ethanol.

\section{RESULTS}

We found that the paper consistently moved towards the magnet. Figure 1 shows a representative series of snapshots of the paper's position over time. The paper moved steadily toward the magnet. As it moved, it tended to rotate. This rotational effect was observed in all experiments, although the direction was not 
consistent: in some trials, the paper rotated clockwise while in others, it rotated counterclockwise. However, movement toward the magnet was consistent in all experiments $(n=4)$
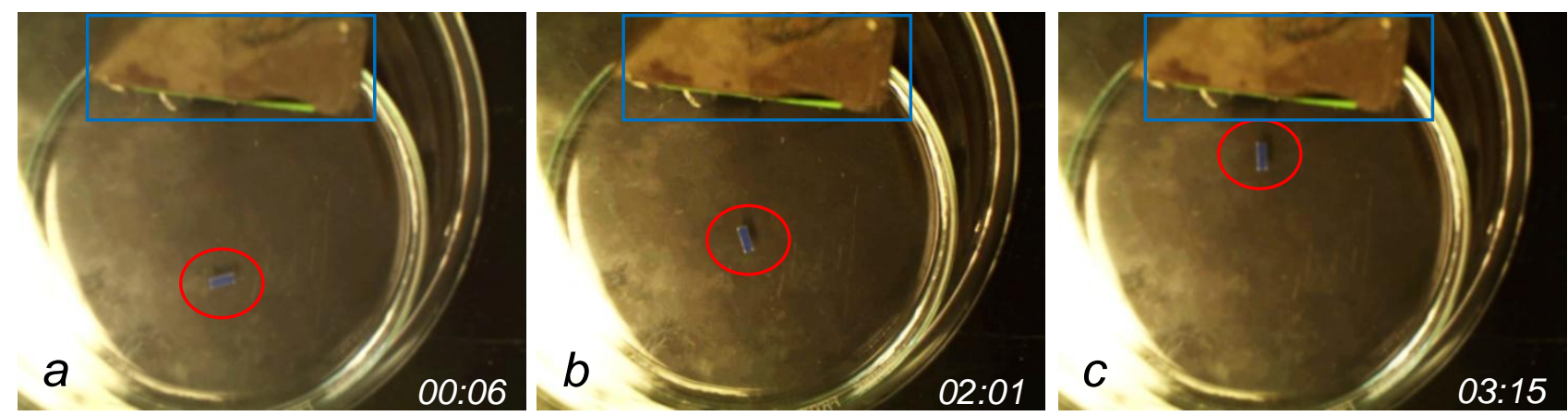

Figure 1. Snapshots of the paper's position over time. The paper strip is highlighted by red circle. The bar at the top, enclosed by the blue rectangle, is the neodymium magnet. The smaller circular container is the petri dish that holds the water and paper, while the larger circular container is the cover. Numbers on lower right indicate elapsed time in minutes.

The paper's distance from the magnet (reckoned from the paper's near edge) was averaged over four experiments and plotted in Figure 2. Velocity increased as the paper approached the magnet, implying a stronger pull as the paper got closer to the magnet. Even though the paper was positioned in roughly the same place initially, the amount of time taken to reach the magnet varied from one experiment to the next, causing the standard deviation to increase over time.

In a control experiment, the magnet was replaced by a 65 $x 19 \times 19 \mathrm{~mm}$ block of brass. Figure 3 shows a plot of the paper's distance-from-metal over a time period similar to that of Figure 2. We found no significant change of position. Hence, the movement observed in Figure 2 was apparently the result of attraction by the magnet.

The effect of increased paper size is shown in Figure 4. When the size increased, the paper took longer to reach the magnet. Although the starting distances were similar within $10 \%$, the required time for the larger paper was approximately 450 seconds compared to 350 seconds for the smaller paper. Hence, the size of the object affects how long it takes to reach the magnet.

Other than the relatively longer time, the position curves (Figs. 2 and 4) appear similar. In both instances, the velocity of the paper increased as the distance between the paper and the magnet diminished. In addition, both sized papers experienced similar rotational motions as they moved toward the magnet. In some trials the paper continuously rotated clockwise, while in others it rotated counterclockwise. When we changed the poles of the

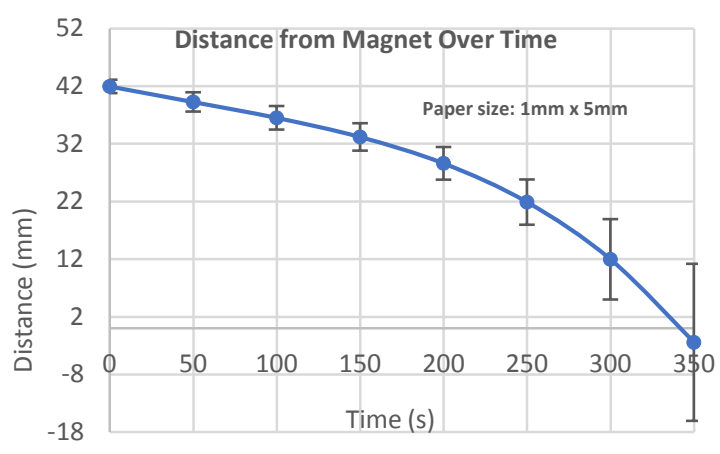

Figure 2. Distance of paper from the magnet over time. $\mathrm{N}=4$. Bars represent SD.

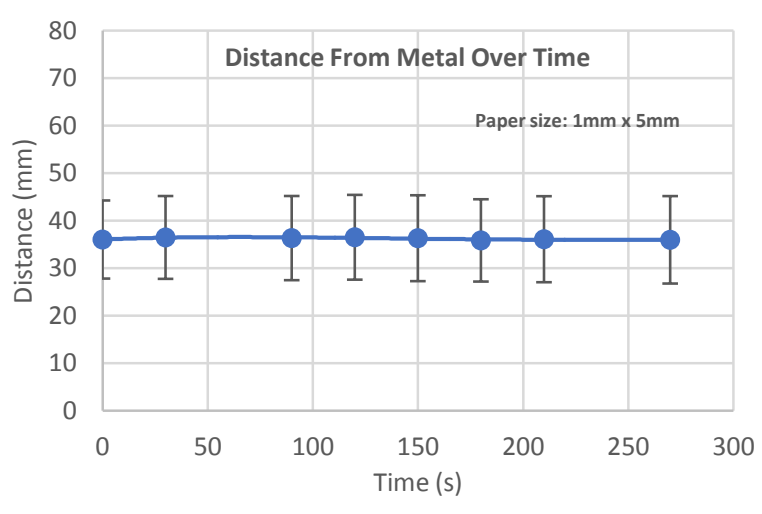

Figure 3. Control experiment. Average distance of paper from metal over time. $\mathrm{N}=3$. The relatively large SD was the result of differences of the papers' initial position. 
magnet and made the north pole face the paper, we observed similar results.

We examined several other buoyant materials to determine whether the effect was exclusive to paper. Samples included Nafion, Teflon, and wood, and the results are shown in Figure 5. In all instances, the objects moved toward the magnet, with dynamics roughly similar to that of paper. The Nafion tube took approximately 650 seconds to move $25 \mathrm{~mm}$, while the Teflon tube and wooden toothpick segment took roughly 210 seconds. Evidently, the attraction by the magnet appears to be rather general.

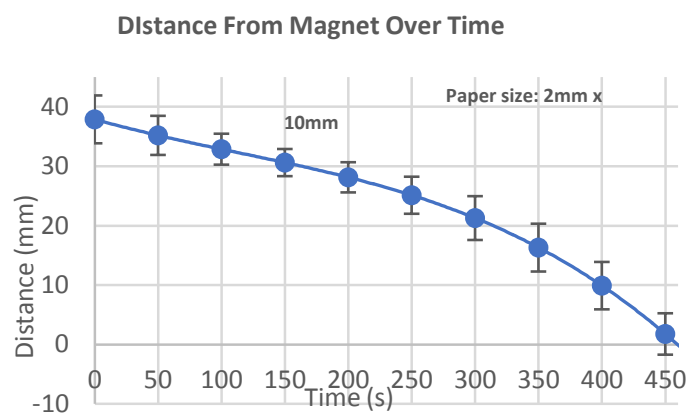

Figure 4. The larger paper's average distance from the magnet over time. $\mathrm{N}=5$
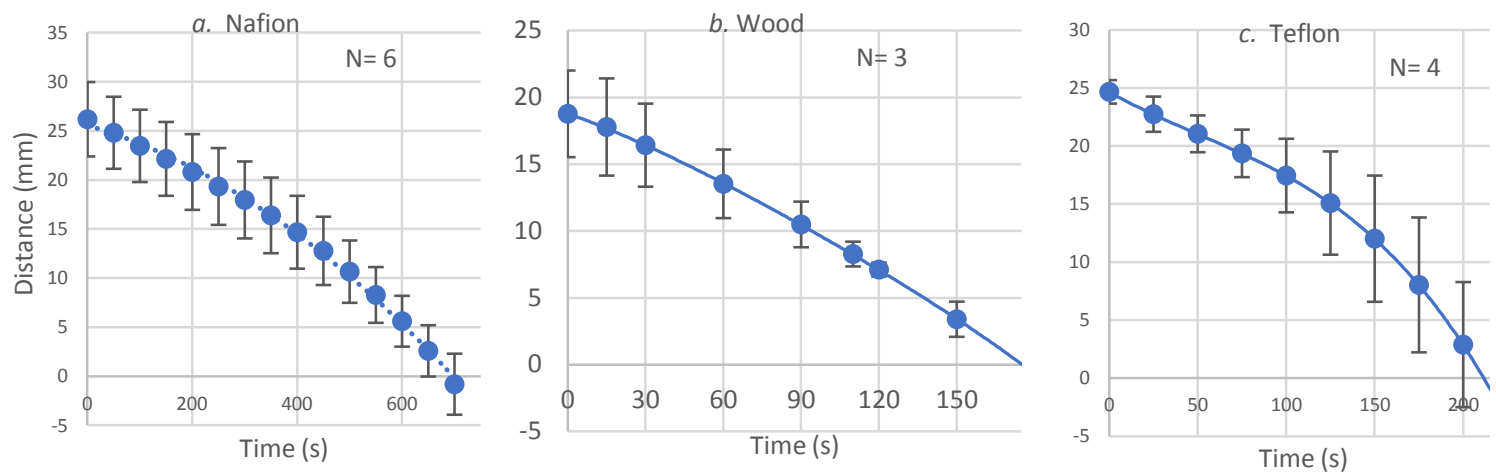

Figure 5. Distance from magnet over time for various materials.
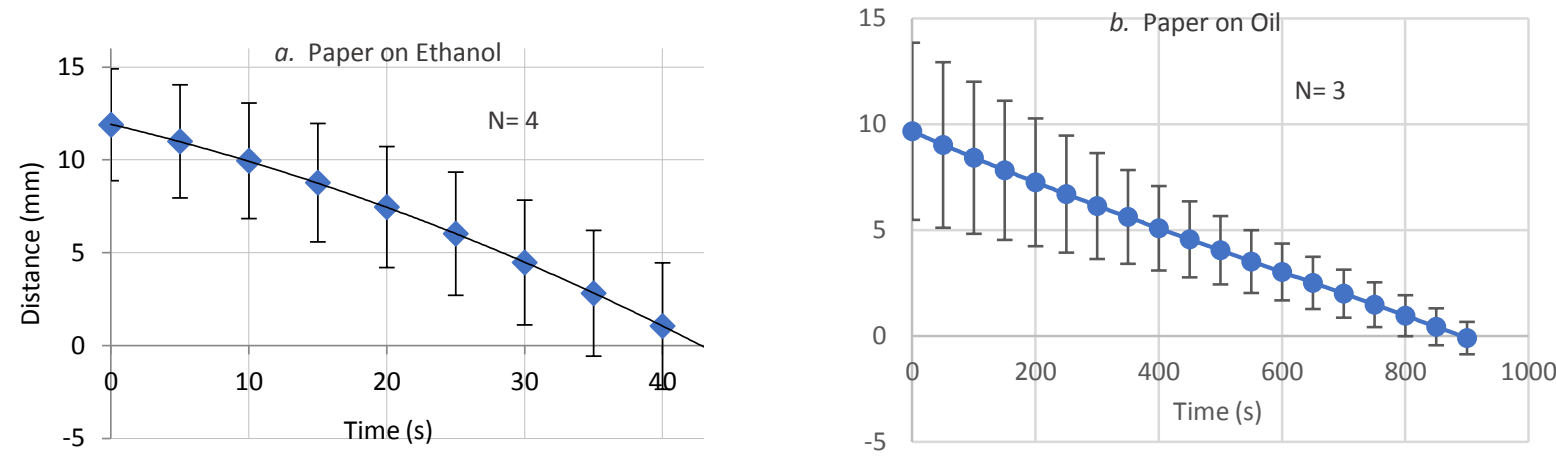

Fiqure 6. Time course of paper's distance from maqnet when placed on ethanol (a) and mineral oil

Finally, we explored whether the presence of the underlying water was critical. To do so, the water was replaced by different liquids. Figure 6 shows the time course of the paper's distance from the magnet when floating on ethanol (a), and mineral oil (b). The paper moved toward the magnet, irrespective of the nature of the liquid. Hence, water is not critical for the movement.

\section{DISCUSSION}

None of the materials used in these experiments are classified as ferromagnetic or paramagnetic. Hence, according to present understanding, those objects should not experience a force when placed in a magnetic 
field. However, when a neodymium magnet was placed nearby, all materials were seen to move towards the magnet.

As shown in Figures 1 and 2, when the paper was floated on water with a magnet nearby, the paper slowly moved towards the magnet. When the magnet was replaced by a nonmagnetic metal bar, the paper's position did not change over time (Figure 3). Since the only difference between the two experiments was the presence/absence of the magnetic field, the outcome implies that the paper moves only when it is placed in a magnetic field.

The results shown in Figure 5 indicate that materials other than paper may experience similar attraction. However, the time taken by the wood, Nafion, and Teflon objects to move towards the magnet differed, despite starting out at roughly the same distance from the magnet. This implies that the magnitude of the force felt by the objects depends on the type of material and perhaps the shape.

The position curves shown in Figures 2, 4, 5, and 6a all show similar character, regardless of the material, size, and medium. The curved shape implies that the velocity increases as it enters a region where the magnetic field is stronger. Only in Figure $6 \mathrm{~b}$, with mineral oil, is the curve not concave downwards, but relatively straight. The absence of acceleration could be due to the oil's high viscosity, which prevented the paper from noticeably accelerating.

Two possible mechanisms may explain why the objects moved towards the magnet. The first hypothesis is that the magnet caused the water to circulate in such a way that the top layer flows towards the magnet, dragging the floated objects along. The second, more direct explanation is that the objects themselves experience an attractive force towards the magnet.

The first hypothesis appears less likely for several reasons: First, all the objects floating on water experienced an acceleration over time. As they got closer to the magnet, their velocities increased. If the water circulating in the petri dish were responsible for the movement, this would imply that the top layer must also accelerate as the object got closer to the magnet. However, the top surface can move towards the magnet only if some other water moves away from the magnet, presumably near the bottom of the petri dish. Since the objects moved faster closer to the magnet, the rate of flow at the bottom of the petri dish must therefore also be faster at areas closer to the magnet, and slower at positions farther away. This would imply that at some points in this dish, the net flow into the point must exceed that flowing out, which violates the principle of continuity [9].

The second argument against the first hypothesis is that even though the same effect was observed when the paper size increased, the time taken for the larger sized paper to reach the magnet was considerably longer than that for the smaller one. If the water were dragging the paper towards the magnet, the larger surface area should have little effect on the speed. However, the larger strip took an average of 100 seconds longer to reach the magnet.

The third reason to favor the second hypothesis over the first is that the same effect was seen when the liquid was changed from water to mineral oil or ethanol. Hence, some unique feature of water could not have been responsible for the movement. Evidently, the floating objects themselves must have felt an attraction towards the magnet.

The final argument is based on the diamagnetic character of water $[8,10,11]$. Since water is slightly diamagnetic, the water should be weakly repelled when placed near a strong magnet. Since the top layer of the water lies closest to the magnet, that water should be moving away from the magnet rather than towards it. Floating objects moving towards the magnet would have to overcome that retarding drag force.

For all the reasons above, we are tempted to conclude that the objects themselves are attracted to the magnet. Hence, many objects considered "non-magnetic" may in fact be magnetic. 


\section{CONCLUSION}

When a strip of paper was floated on water, the paper moved towards the nearby magnet, and sped up as it got closer. Doubling the size of the paper lengthened the time it took for the paper to reach the magnet. This attraction was also observed in other materials including Teflon, Nafion, and wood; and it was seen as well when the suspending medium was replaced by other liquids. Hence, the attraction effect appears to be direct. Possibly, all materials are magnetic to some degree.

\section{REFERENCES}

1. P. O'Grady, "Thales of Miletus (c. 620 B.C.E.-C. 546 B.C.E.)," in Internet Encyclopedia of Philosophy. Internet Encyclopedia of Philosophy. [Online]. Available: http://www.iep.utm.edu/thales/

2. J. Vardalas, "A History of the Magnetic Compass," The Institute IEEE. [Online]. Available: http://theinstitute.ieee.org/tech-history/technology-history/a-history-of-the-magnetic-compass

3. H. T. Masood, Z. Muhammad, M. Habib, D. Wang and D. Wang, "Low temperature ferromagnetic properties of CdS and CdTe thin films," Chinese Physics B, vol. 26, no. 6, 2017. [Online]. Available: http://iopscience.iop.org/article/10.1088/1674-1056/26/6/067503

4. Y.W. Ma et al., "Room temperature ferromagnetism in Teflon due to carbon dangling bonds," Nature Communications, vol. 3, no. 727, Mar. 2012. [Online]. Available: https://www.nature.com/articles/ncomms1689

5. D.L. Sage et al., "Optical magnetic imaging of living cells," Nature, vol. 496, pp. 486-489, Apr. 2013. [Online]. Available: https://www.nature.com/articles/nature12072

6. M. Zehetmayer et al., "Simulation of the current dynamics in a superconductor induced by a small permanent magnet: application to the magnetoscan technique," Superconductor Science and Technology, vol. 19, no. 7, pp. S429-S437, Apr. 2006. [Online]. Available: http://iopscience.iop.org/article/10.1088/0953-2048/19/7/S02

7. B.P. Mann and N.D. Simms, "Energy harvesting from the nonlinear oscillations of magnetic levitation," Journal of Sound and Vibration, vol. 319, no. 1-2, pp. 515-530, Jan. 2009. [Online]. Available: https://www.sciencedirect.com/science/article/pii/S0022460X08005567

8. B.M. Moskowitz, "Hitchhiker's Guide to Magnetism," Institute for Rock Magnetism, University of Minnesota, 1991. [Online]. Available: http://www.irm.umn.edu/hg2m/hg2m.pdf

9. Y. Cengel and J Cimbala. "Fluid Mechanics Fundamental and Applications," New York, NY: The McGraw-Hill Companies Inc, 2014.

10. J. S. Philo and W. M. Fairbank, "Temperature dependence of the diamagnetism of water," Journal of Chemical Physics, vol. 72, no. 8, pp. 4429-4433, 1980. [Online]. Available: https://aip.scitation.org/doi/abs/10.1063/1.439734

11. R. Cini and M. Torrini, "Temperature Dependence of the Magnetic Susceptibility of Water," Journal of Chemical Physics, vol. 49, no. 6, pp. 2826, 1968. [Online]. Available: https://aip.scitation.org/doi/abs/10.1063/1.1670491 